ournal for ImmunoTherapy of Cancer

\title{
Atezolizumab in patients with renal insufficiency and mixed variant histology: analyses from an expanded access program in platinum-treated locally advanced or metastatic urothelial carcinoma
}

\author{
Jean Hoffman-Censits, ${ }^{1}$ Sumanta Pal, ${ }^{2}$ Constanze Kaiser, ${ }^{3}$ Beiying Ding, ${ }^{3}$ \\ Joaquim Bellmunt ${ }^{4}$
}

To cite: Hoffman-Censits J, Pal S, Kaiser C, et al. Atezolizumab in patients with renal insufficiency and mixed variant histology: analyses from an expanded access program in platinum-treated locally advanced or metastatic urothelial carcinoma. Journal for ImmunoTherapy of Cancer 2020;8:e000419. doi:10.1136/ jitc-2019-000419

Accepted 04 May 2020

\section{Check for updates}

(c) Author(s) (or their employer(s)) 2020. Re-use permitted under CC BY-NC. No commercial re-use. See rights and permissions. Published by BMJ.

${ }^{1}$ Departments of Oncology and Urology, Johns Hopkins Sidney Kimmel Comprehensive Cancer Center, Baltimore, MD, USA, Baltimore, Maryland, USA ${ }^{2}$ Department of Medical Oncology \& Experimental Therapeutics, City of Hope Comprehensive Cancer Center, Duarte, California, USA ${ }^{3}$ Genentech Inc, South San Francisco, California, USA ${ }^{4}$ Director, Bladder Cancer Program, Beth Israel Deaconess Medical Center, Boston, Massachusetts, USA

Correspondence to Dr Jean Hoffman-Censits; jhoffm57@jhmi.edu

\section{ABSTRACT}

Background Atezolizumab is a treatment for locally advanced/metastatic urothelial carcinoma (mUC). However its use in patients with renal insufficiency or UC with mixed variant histology $(\mathrm{MVH})$ is not well characterized.

Objective To report efficacy and safety of atezolizumab in these special subpopulations from an expanded access program (EAP).

Design, setting, and participants A total of 218 patients were enrolled at 36 US study sites (November 2015-August 2016), and the trial ended following the approval of atezolizumab by the US Food and Drug Administration. This post hoc analysis investigated outcomes in specific study subgroups.

Intervention Atezolizumab 1200 mg was administered intravenously every 3 weeks until loss of clinical benefit, unacceptable toxicity, death, consent withdrawal, decision to discontinue, commercial availability, or study closure. Outcome measurements and statistical analysis Response Evaluation Criteria in Solid Tumors V.1.1 responses and safety were evaluated by baseline renal function and histology.

Results and limitations Objective responses occurred in $0 / 6(0 \%), 4 / 19(21 \%), 1 / 27(3.7 \%)$, and $12 / 62(19 \%)$ of evaluable patients with creatinine clearance $(\mathrm{CrCl})<30$, $30-45,45-60$, and $\geq 60 \mathrm{~mL} / \mathrm{min}$, respectively, and stable disease was seen in three patients with $\mathrm{CrCl}<30 \mathrm{~mL} /$ min. Objective responses were seen in 13/102 patients (13\%) with urothelial carcinoma (UC) histology only and in 4/12 patients (33\%) with UC with MVH. Treatmentrelated adverse event frequencies ranged from $35 \%$ to $54 \%$ across the earlier indicated $\mathrm{CrCl}$ subgroups and they were also similar in patients with pure UC or UC with MVH (46\%).

Conclusions In this EAP mUC subgroup analysis, clinical benefit of atezolizumab occurred in patients with compromised renal function or MVH UC tumors. Safety was comparable across subgroups.

Patient summary We examined the efficacy and safety of atezolizumab for UC in certain patients participating in an EAP. We found that responses to atezolizumab occurred, and safety was similar, in most patient subgroups with varying levels of kidney functioning or less common types of tumor tissue histology.

\section{INTRODUCTION}

Locally advanced or metastatic urothelial carcinoma (mUC) is often treated with cisplatin-based chemotherapy as a standard first-line approach. ${ }^{12}$ However, many patients are cisplatin-ineligible, and carboplatinbased alternatives are associated with shorter overall survival (OS). ${ }^{3}$ Most patients eventually progress following platinum-based regimens. ${ }^{4}$ Evidence shows that non-treatment is common $^{125}$ and 5-year survival rates in the USA have historically been low. ${ }^{6}$ Recently, checkpoint inhibitors have been approved in the USA, Europe, and elsewhere for patients with mUC who experience progression during or following platinum-based chemotherapy. ${ }^{7} 8$ Prior to atezolizumab's US approval, and based on favorable data from earlier studies, ${ }^{9-11}$ an expanded access program (EAP) was conducted to grant access to this patient population. This $\mathrm{EAP}^{12}$ enrolled a broader range of patients than those who are typically enrolled in phase 1-3 clinical trials, providing an opportunity to better understand the role of atezolizumab in special populations.

In patients with mUC, baseline clinical characteristics or pre-existing comorbidities can limit treatment options and lead to treatment complications. One condition that is common to many patients with $\mathrm{mUC}$ is renal insufficiency (eg, creatinine clearance $(\mathrm{CrCl})$ of $<60 \mathrm{~mL} / \mathrm{min}$ ), which may be due to factors such as age, smoking-related vascular disease, 
urothelial carcinoma (UC)-related urinary tract obstruction, ${ }^{5}$ recent nephroureterectomy, ${ }^{13}$ and postcystectomy hydronephrosis. ${ }^{14}$ Renal insufficiency can preclude firstline cisplatin administration ${ }^{5}$ or stem from treatment with cisplatin. ${ }^{15}$ Since renal function declines steadily after age $30^{16}$ and the median age at UC diagnosis in the USA is 72 years, significant decline in renal function is not uncommon among newly diagnosed patients, ${ }^{56}$ as demonstrated in a study of patients undergoing radical cystectomy, wherein $>40 \%$ of patients older than 70 years had a $\mathrm{CrCl}$ of $<60 \mathrm{~mL} / \mathrm{min} .{ }^{17}$ One meta-analysis with data from $>5000$ UC cases demonstrated a correlation between preoperative renal insufficiency and worse prognosis, underscoring the importance of renal function in developing an optimal treatment plan. ${ }^{18}$

Another consideration is that although the most common histological type of urinary tract cancers is UC, with bladder being the most common location (followed by renal pelvis, ureter, and urethra) ${ }^{19} \mathrm{UC}$ with mixed histological features, including micropapillary, glandular, squamous, sarcomatoid, plasmacytoid, lymphoepithelioma like, and/or nested histology components, as defined by the WHO, ${ }^{19}$ is quite common. In cystectomy specimens, the incidence of $\mathrm{UC}$ with mixed variant histology (MVH; sometimes termed divergent differentiation) may reach $33 \% .{ }^{19}$ Data suggested that some variants of pure non-UC (eg, small-cell and squamous bladder cancer) portend worse prognosis than UC and should be treated differently, but whether these results extend to all patients with MVH remains less well characterized. ${ }^{20}$ Although MVH appears to be associated with predictors of tumor aggressive behavior and adverse outcome in some studies, outcome data are limited, ${ }^{19}{ }^{21}$ with occasional case reports suggesting that immunotherapy is active. ${ }^{22}$ In this analysis from the EAP study, we evaluated efficacy and safety outcomes with atezolizumab in patients with impaired baseline renal function or UC with MVH.

\section{PATIENTS AND METHODS}

\section{Study design, patients, and procedures}

The overall study design and primary analysis of this single-arm, open-label EAP were previously reported ${ }^{12}$ ( ClinicalTrials.gov ID NCT02589717). The primary objective was to provide access to atezolizumab to eligible patients before US Food and Drug Administration (FDA) approval was granted.

Key eligibility criteria included histologically or cytologically documented, inoperable, locally advanced (T4b, any $\mathrm{N}$ or any $\mathrm{T}, \mathrm{N} 2-3$ ), or metastatic (M1 or stage IV) $\mathrm{UC}$; primary tumor site of bladder, urethra, renal pelvis, or ureter with dominant UC (transitional cell) histology; Eastern Cooperative Oncology Group performance status (ECOG PS) of 0-2; and disease recurrence or progression during or following $\geq 1$ platinum-containing regimen ( $\geq 2$ cycles) for mUC. Patients who progressed within 12 months of treatment with a platinum-containing adjuvant/neoadjuvant regimen were also eligible. The number of prior therapies a patient could have received was unrestricted. Enrollment was initially restricted to programmed death ligand 1 (PD-L1)-selected patients (tumor-infiltrating immune cell 2/3 (IC2/3) status and PD-L1 expression on IC of $\geq 5 \%$ per VENTANA SP142 PD-L1 immunohistochemistry assay) but was later extended per protocol amendment to allow enrollment regardless of PD-L1 status. Enrollment also initially excluded patients with significant renal disorders requiring dialysis or indications for renal transplant but it was later amended such that there were no restrictions on $\mathrm{CrCl}$ levels.

Patients received atezolizumab $1200 \mathrm{mg}$ intravenously every 3 weeks and they could be treated past disease progression (evaluated per Response Evaluation Criteria in Solid Tumors V.1.1 (RECIST V.1.1)) or until loss of clinical benefit (per investigator), unacceptable toxicity, consent withdrawal, decision to discontinue treatment (per patient or physician), death, commercial study drug availability (following FDA approval), or study termination. Tumor assessments occurred approximately every 9 weeks (every 12 weeks after the first 54 weeks).

\section{Study assessments and analyses}

Key efficacy objectives included investigator-assessed RECIST V.1.1 objective response rate (ORR) and disease control rate (DCR) (frequency of patients with complete response (CR), partial response (PR), or stable disease $(\mathrm{SD}))$. Response-evaluable patients were those with measurable disease at baseline and $\geq 1$ non-missing postbaseline tumor assessment. OS was defined as the time from study treatment initiation to death from any cause. Safety was assessed per Common Terminology Criteria for Adverse Events V.4.0. Safety-evaluable patients were those who received $\geq 1$ dose of atezolizumab. Follow-up was defined as the duration between initiation of atezolizumab and when the patient was last known to be alive or had died.

Post hoc subgroup analyses of efficacy and safety were performed based on baseline $\mathrm{CrCl}$ levels $(<30,30-45$, $45-60$, and $\geq 60 \mathrm{~mL} / \mathrm{min}$ ) and tumor histology (pure UC histology only or UC with MVH). There was no formal statistical hypothesis testing, and all analyses were descriptive.

\section{RESULTS \\ Patients and treatment}

This study enrolled 218 patients from 36 US study sites between November 2015 and August 2016, ending on FDA approval of atezolizumab. Patients could continue on commercially available atezolizumab treatment as appropriate, but study assessments aside from optional long-term survival follow-up did not continue. The data cut-off was October 5, 2016, at which time the median follow-up in all treated patients was 2.3 months (range 0.03-6.7). In the overall cohort, 214 patients were treated and were evaluable for safety, and 114 were evaluable for response assessments (figure 1). Overall, among treated 


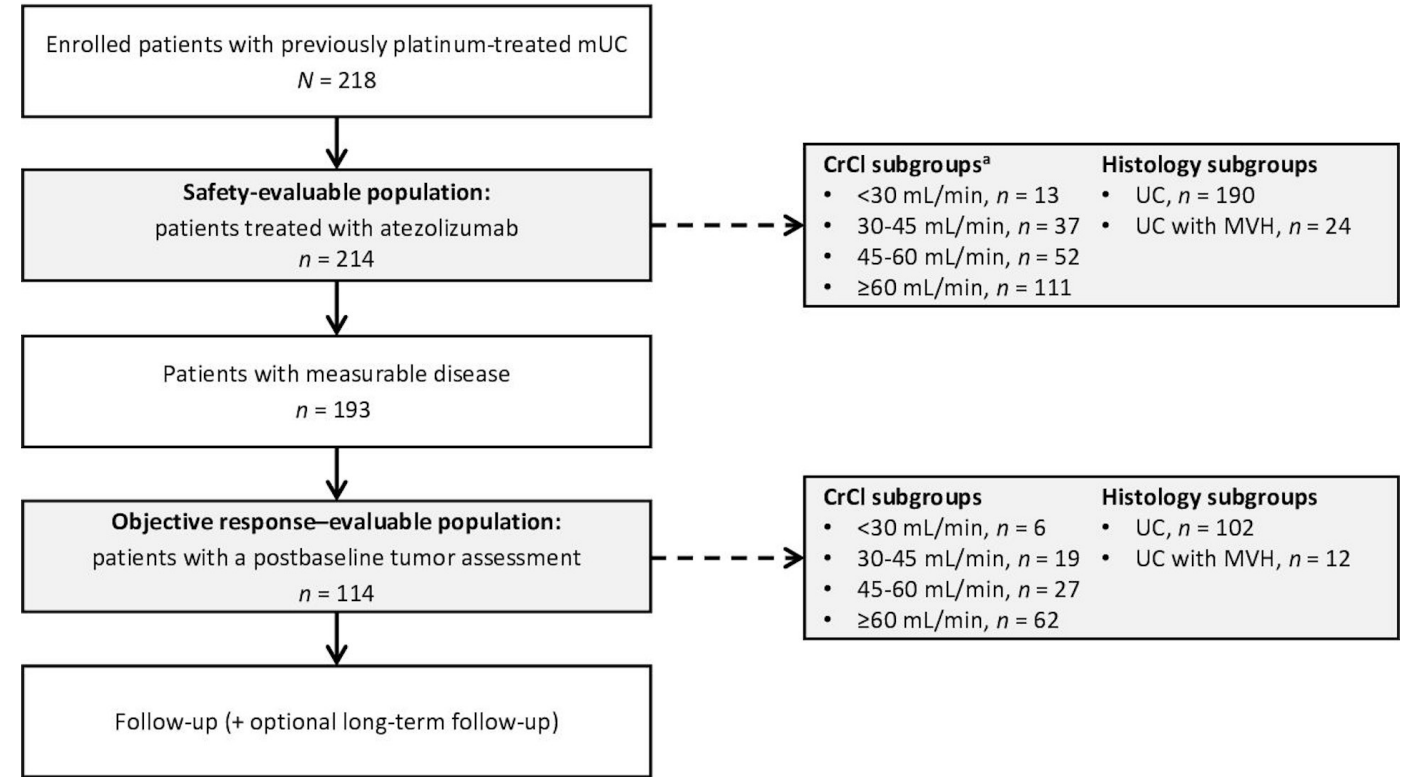

Figure 1 Patient flow. Patients (enrolled between November 2015 and August 2016), efficacy and safety-evaluable populations, and $\mathrm{CrCl}$ and histology analysis subgroups are shown. ${ }^{a}$ One patient had missing $\mathrm{CrCl}$ status. $\mathrm{CrCl}$, creatinine clearance; mUC, metastatic urothelial carcinoma; $\mathrm{MVH}$, mixed variant histology; UC, urothelial carcinoma.

patients, characteristics were balanced across subgroups of interest (table 1) with a few exceptions. Fewer men were in the two lowest $\mathrm{CrCl}$ subgroups. The lowest $\mathrm{CrCl}$ subgroup and the MVH group contained fewer patients with ECOG PS of 0. Among evaluable patients with $\mathrm{CrCl}$ of $<30 \mathrm{~mL} / \mathrm{min}$ and non-missing PD-L1 status, all had IC2/3 $(n=5)$.

\section{Efficacy}

In the overall cohort of objective response-evaluable patients $(\mathrm{n}=114)$, the ORR was $15 \%(95 \% \mathrm{CI}, 9 \%$ to $23 \%)$ and the DCR was $49 \%$ (95\% CI, $40 \%$ to $59 \%$ ). Objective responses occurred in patient subgroups with baseline $\mathrm{CrCl}$ of $\geq 30 \mathrm{~mL} / \mathrm{min}$ : in 4 patients with $30-45 \mathrm{~mL} /$ $\min (21 \%), 1$ with $45-60 \mathrm{~mL} / \mathrm{min}(4 \%)$, and 12 with $\geq 60 \mathrm{~mL} / \mathrm{min}(19 \%)$. CRs occurred in one patient with $\mathrm{CrCl}$ of $30-45 \mathrm{~mL} / \mathrm{min}$ and two patients with $\mathrm{CrCl}$ of $\geq 60 \mathrm{~mL} / \mathrm{min}$. Additionally, three of the six patients with $\mathrm{CrCl}$ of $<30 \mathrm{~mL} / \mathrm{min}$ achieved SD (figure 2 ). Due to the short duration of the study with a median follow-up of 2.3 months, OS data were immature. At the time of the analysis, $23 \%-31 \%$ had died across $\mathrm{CrCl}$ subgroups.

The ORR was $13 \%$ in patients with pure UC only $(n=13$ of 102$)$ and $33 \%$ in patients with UC with MVH $(n=4$ of 12$)$. Eleven patients with pure UC had PR, and two had CR. Responses did not appear to cluster with specific mixed histology components (figure 2); three PRs-in one of the two patients with an adenocarcinoma histology component, in a patient with a glandular component, and in a patient with a component with sarcomatoid/rhabdoid differentiation-and 1 CR occurred (in a patient with UC with MVH with squamous/glandular differentiation). SD as best response also occurred in 36 patients (35\%) in the pure UC population and in $3(25 \%)$ of the UC with MVH population, the latter of which included those with squamous $(n=2)$ or papillary $(\mathrm{n}=1)$ histology components. The DCRs were $48 \%$ and $58 \%$ in patients with pure UC and UC with MVH, respectively (figure 2).

\section{Safety}

All-grade treatment-related adverse events (TRAEs) occurred in 98 of the 214 safety-evaluable patients $(46 \%)$ and ranged from $35 \%$ (in the $\mathrm{CrCl} 30-45 \mathrm{~mL} /$ min subgroup) to $54 \%(\mathrm{CrCl} 45-60 \mathrm{~mL} / \mathrm{min})$. Overall, 11 patients $(5.1 \%)$ had serious TRAEs that occurred in two patients with $\mathrm{CrCl}$ of $<30 \mathrm{~mL} / \mathrm{min}(15 \%)$ and at rates of $\leq 8 \%$ in other subgroups. Four patients $(2 \%)$ had TRAEs leading to treatment discontinuation $(\leq 3 \%$ across subgroups), and none were due to renal events. Overall, 59 patients (28\%) had AEs of special interest (AESIs), and AESI rates ranged from $15 \%(\mathrm{CrCl}$ $<30 \mathrm{~mL} / \mathrm{min})$ to $32 \%(\mathrm{CrCl} \geq 60 \mathrm{~mL} / \mathrm{min})$ (table 2$)$. Renal treatment-related AEs occurred only in patients with baseline $\mathrm{CrCl}$ of $\geq 30 \mathrm{~mL} / \mathrm{min}$, including immunerelated nephritis $(n=2)$, acute kidney injury $(n=2)$, and proteinuria $(\mathrm{n}=1)$.

TRAEs occurred in 87 patients with pure UC and 11 patients with UC with MVH tumors (46\% each). Four patients with pure UC $(2.1 \%)$ and none with UC with MVH had TRAEs leading to discontinuation. The frequency of AESIs was also similar between pure UC and UC with MVH subpopulations (27\% and 29\%, respectively). AESIs were not observed in patients with UC mixed with adenocarcinoma or sarcomatoid histology but they occurred in 1 of the 8 patients (13\%) with UC mixed with squamous histology and 6 of the 10 patients $(60 \%)$ with other MVH components (table 2). 


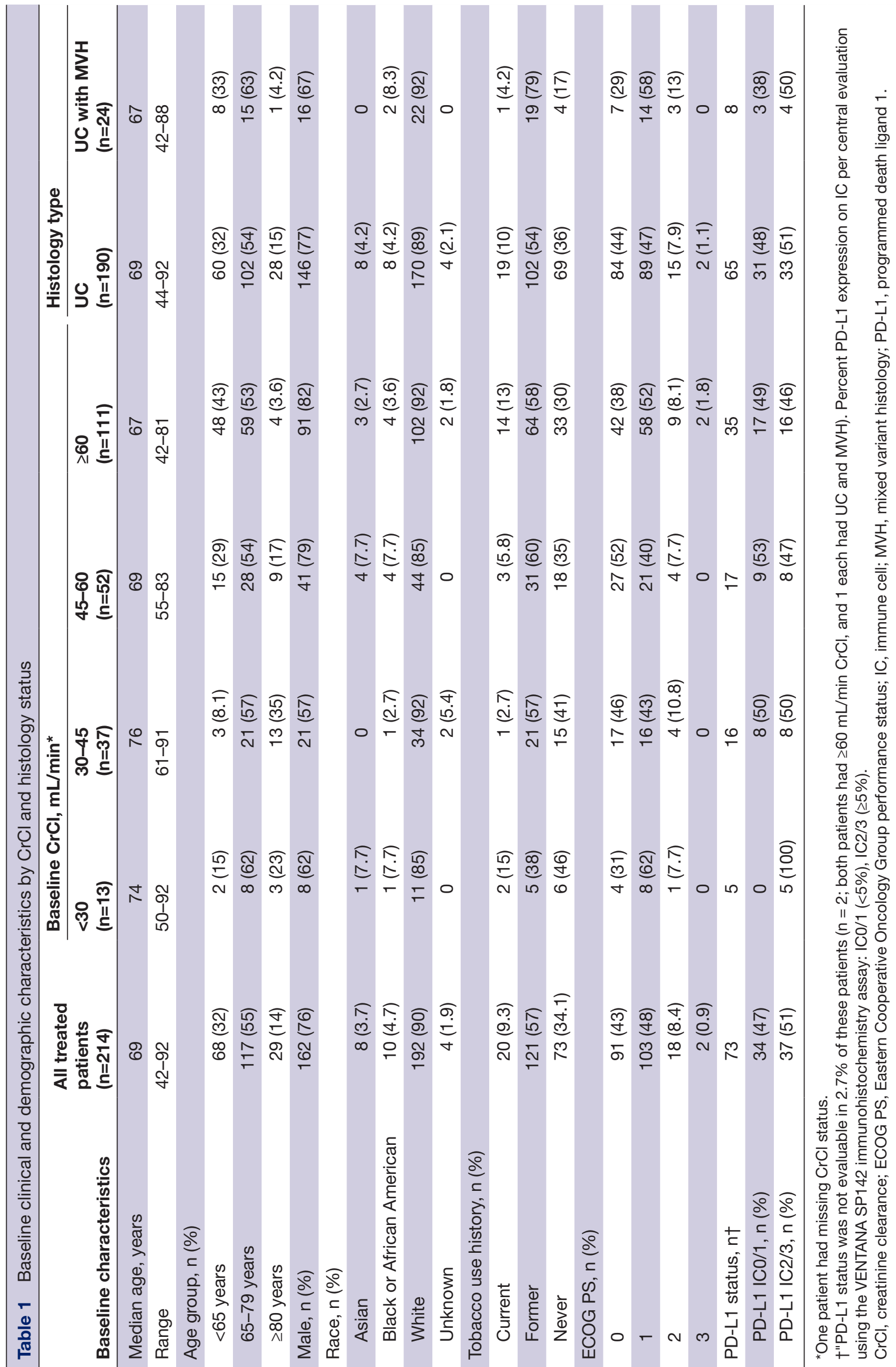




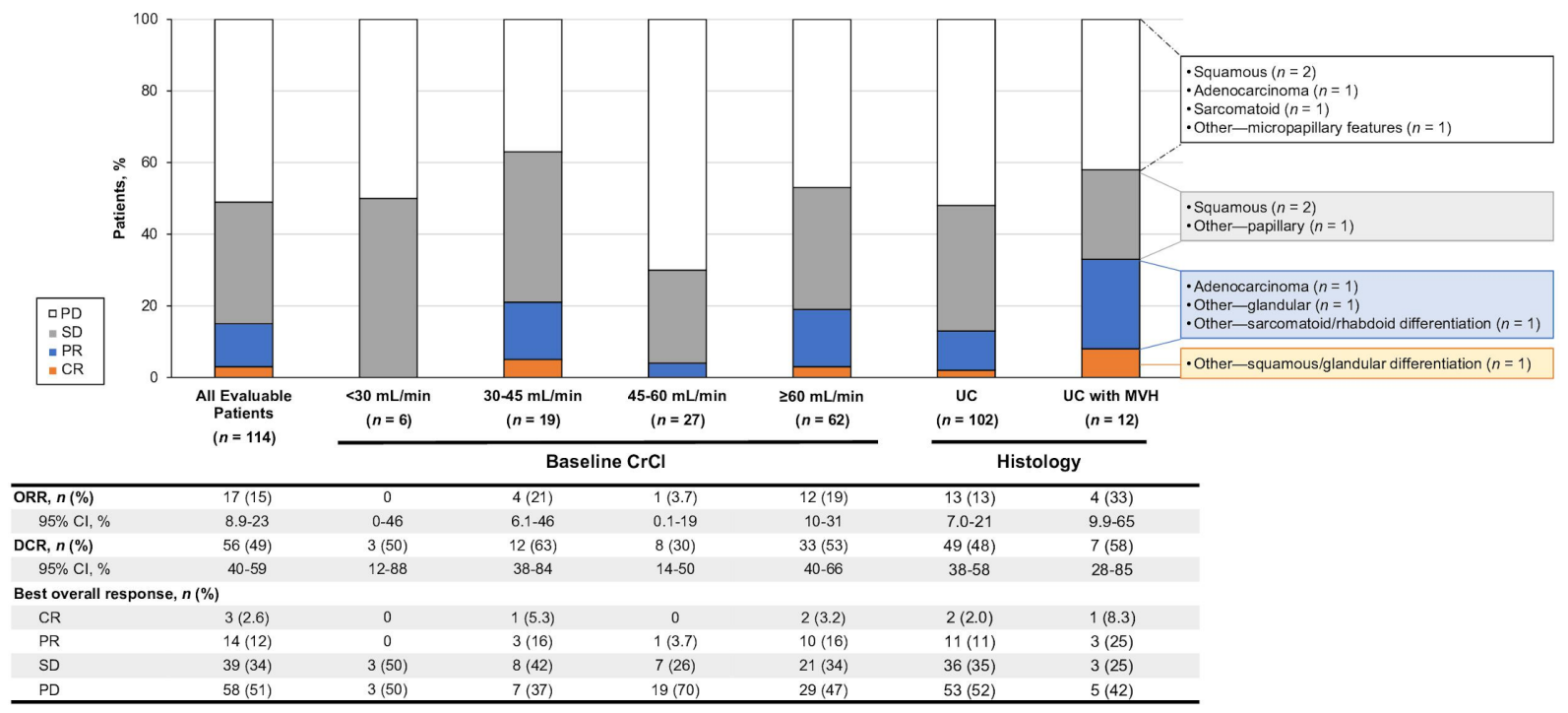

Figure 2 Response by CrCl levels and histology subtype. RECIST V.1.1 ORR and DCR and best overall response are shown by $\mathrm{CrCl}$ levels and by histological subtype. $\mathrm{CR}$, complete response; $\mathrm{CrCl}$, creatinine clearance; $\mathrm{DCR}$, disease control rate; MVH, mixed variant histology; ORR, objective response rate; PD, progressive disease; PR, partial response; RECIST, Response Evaluation Criteria in Solid Tumors; SD, stable disease; UC, urothelial carcinoma.

\section{DISCUSSION}

The availability of checkpoint inhibitors such as atezolizumab has greatly changed the treatment landscape for patients with $\mathrm{UC}$, including those who progress on platinum-based chemotherapy for whom treatment options were previously limited to chemotherapy. However, there still exists an unmet need for patients with comorbidities or uncommon tumor features-who are often excluded, under-represented, or not comprehensively evaluated in clinical trials-resulting in limited outcomes data for these subsets. In particular, patients with renal insufficiency or non-UC histology may have worse prognoses than their counterparts. ${ }^{1821}$ Following a protocol amendment, this EAP allowed for enrollment of patients irrespective of $\mathrm{CrCl}$ levels or the presence of renal disorders requiring dialysis or indications for renal transplant, permitting characterization of patients not eligible for the pivotal phase 2-3 IMvigor210 and IMvigor211 studies (atezolizumab in platinum-treated mUC). Furthermore, this EAP reported an $11 \%$ frequency $(\mathrm{n}=24)$ of treated patients with UC with MVH tumors. ${ }^{12}$ This post hoc analysis aimed to evaluate outcomes with checkpoint inhibitor therapy in these subsets of patients.

In this study, objective response occurred in patient subgroups with $\mathrm{CrCl}$ ranging from 30 to $60 \mathrm{~mL} / \mathrm{min}$. These observations are in agreement with data from both the cisplatin-ineligible and platinum-treated cohorts of the IMvigor210 trial, which reported objective responses to atezolizumab in patient subsets with baseline $\mathrm{CrCl}$ of $30-60 \mathrm{~mL} / \mathrm{min}^{3}{ }^{310} \mathrm{SD}$ also occurred in three of the six patients with $\mathrm{CrCl}$ of $<30 \mathrm{~mL} / \mathrm{min}$, a subgroup not included in IMvigor210. These observations also agreed with data from the large, international phase $3 \mathrm{~b}$ SAUL study (atezolizumab in mUC populations broader than/ not eligible for IMvigor211), which found that patients with renal impairment (calculated $\mathrm{CrCl}<30$ but $\geq 15 \mathrm{~mL}$ / min) generally demonstrated ORRs consistent with those of the overall population, and SAUL also demonstrated OS in line with that of previous pivotal UC trials. ${ }^{23}$ In this EAP, responses and SD also occurred in patients with UC with MVH. One patient with other MVH (UC with squamous/glandular differentiation) achieved a CR, and two of the four evaluable patients with UC with a squamous MVH component achieved SD. Furthermore, one of the two evaluable patients with UC mixed with an adenocarcinoma histology component had a PR, and additional instances of PR $(n=2)$ and SD $(n=1)$ in the remaining population were distributed among patients with other mixed histology components (figure 2). Similarly, in the SAUL study that enrolled patients with mixed or non-UC histology, objective responses, including a CR, were also observed. $^{23}$

The safety of atezolizumab was comparable among the subgroups evaluated in this study. Across all evaluated $\mathrm{CrCl}$ subgroups, the frequency of TRAEs was generally comparable, overall ranging from $35 \%$ to $54 \%$. Serious TRAEs occurred in two patients with $\mathrm{CrCl}$ of $<30 \mathrm{~mL} / \mathrm{min}(15 \%)$ and at rates of $\leq 8 \%$ in other subgroups. Renal TRAEs were infrequent, did not lead to discontinuation, and did not occur in patients with $\mathrm{CrCl}$ of $<30 \mathrm{~mL} / \mathrm{min}$. These observations add to data from other studies, including the cisplatin-ineligible cohort of IMvigor210 (wherein atezolizumab was tolerable and did not result in decrease of median glomerular filtration rate in a patient cohort that mostly had renal impairment $(30-60 \mathrm{~mL} / \mathrm{min}))^{3}$ and renally impaired patients from SAUL (in whom safety was similar to that in the overall population) ${ }^{23}$ Also mirroring these safety results from SAUL, ${ }^{23}$ TRAEs in the histology subgroups of this EAP were similar, irrespective of histological features. 


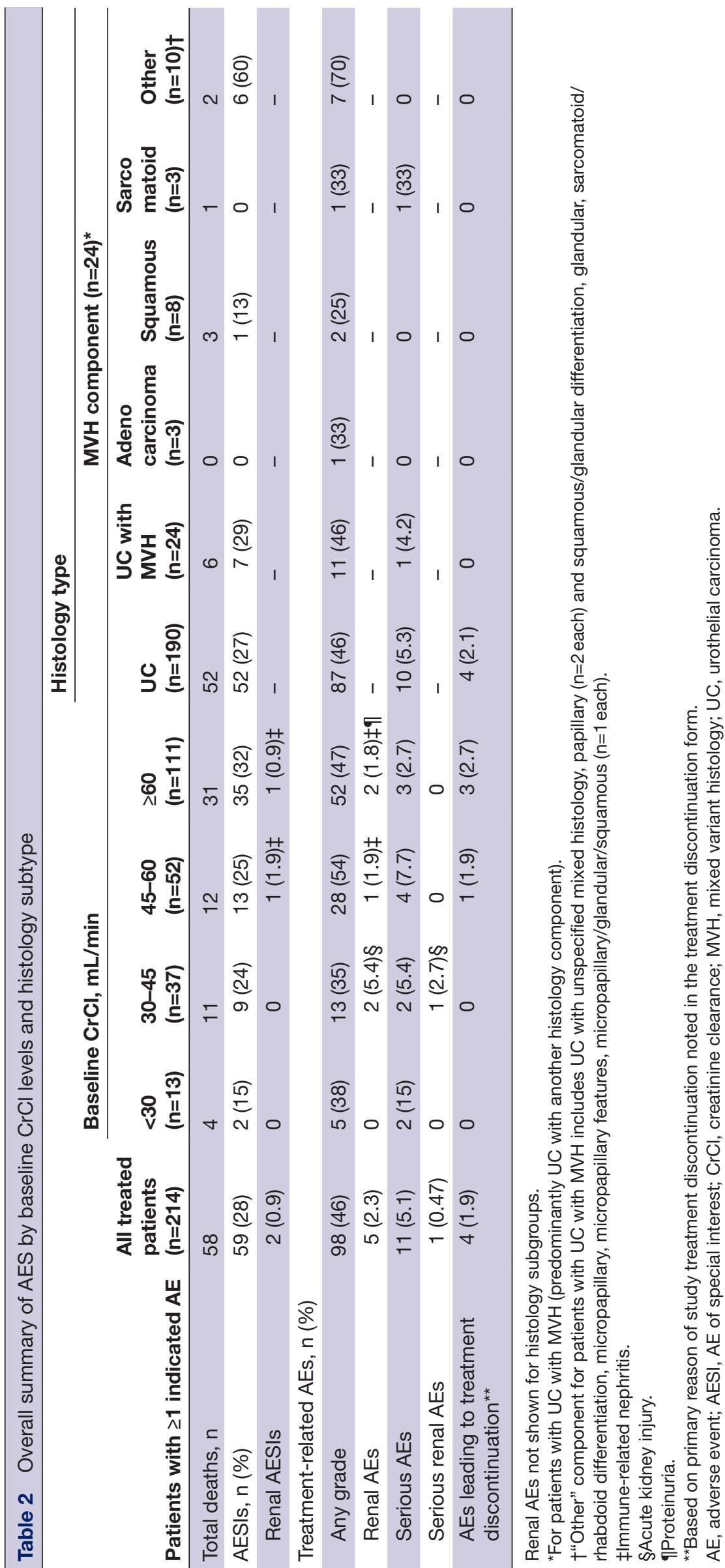


This study provides new information on outcomes with checkpoint inhibitor therapy in special populations of patients with mUC from an EAP, particularly those with $\mathrm{CrCl}$ of $<30 \mathrm{~mL} / \mathrm{min}$, who have often been excluded from clinical trials. These results complement retrospective data from a recent multicenter analysis of patients with advanced solid tumors and baseline organ dysfunction (including renal) treated with anti-programmed death 1 checkpoint inhibitor therapy, ${ }^{24}$ which reported similar rates of immune-related $\mathrm{AE}$ frequencies as those in more general populations and encouraging efficacy, noting that such agents may be a feasible treatment for this challenging patient population. These data also echo recent results from a study on previously platinum-treated patients given the checkpoint inhibitor pembrolizumab, which reported OS benefit in favor of pembrolizumab over chemotherapy, notably for patients with MVH (although safety data were not reported by histological type).${ }^{25} \mathrm{~A}$ limitation of this EAP study was its short duration, based on the timing of atezolizumab approval, ${ }^{12}$ which could affect characterization of both efficacy and safety results. Also, sample sizes in certain subsets were small; thus, current results need to be interpreted with caution, and further analyses in larger populations are warranted. Furthermore, minor differences in baseline characteristics between the subgroups (eg, ECOG PS, PD-L1 status) in this study were not controlled for. Still, these efficacy and safety results suggest that atezolizumab provides clinical benefit to a spectrum of patients with platinum-treated mUC, and these results were recently corroborated by findings in similar patient subgroups (eg, with MVH or renal insufficiency) in a more realworld-like population from the SAUL study. ${ }^{23}$

\section{CONCLUSIONS}

In this subgroup analysis from a US EAP of atezolizumab in patients with previously platinum-treated mUC, outcomes were evaluated by patient baseline renal function and histology. Efficacy and safety were demonstrated in patients with compromised renal function or UC with MVH. The results suggest that atezolizumab provides clinical benefit in a spectrum of patients with platinumtreated mUC.

\footnotetext{
Acknowledgements We thank the patients, their families, and the clinical study site investigators and staff. We thank Darren Tayama, Yong Wang, and Jeremy Linsenmeier for their contributions to the analysis. We thank Qian (Cindy) Zhu for her contributions to the manuscript review. Medical writing assistance for this manuscript was provided by Ashley Pratt, PhD, of Health Interactions, Inc, funded by F. Hoffmann-La Roche, Ltd.
}

Contributors All authors had full access to all of the data in the study and take responsibility for the integrity of the data and the accuracy of the data analysis. Study concept and design: JHC, SP, CK, BD, JB. Acquisition of data: JHC, SP, $\mathrm{BD}, \mathrm{JB}$. Analysis and interpretation of data: JHC, SP, CK, BD, JB. Drafting of the manuscript: $\mathrm{SP}, \mathrm{BD}, \mathrm{CK}$. Critical revision of the manuscript for important intellectual content: JHC, SP, CK, BD, JB. Statistical analysis: SP, BD. Obtaining funding: N/A. Administrative, technical, or material support: SP, JB. Supervision: JB. Other: N/A

Funding F. Hoffmann-La Roche, Ltd sponsored the study and was involved in the design and conduct of the study; management, analysis and interpretation of the data; and preparation, review, and approval of the manuscript. Medical writing assistance was funded by F. Hoffmann-La Roche, Ltd.

Competing interests $\mathrm{JHC}$ certifies that all conflicts of interest, including specific financial interests and relationships and affiliations relevant to the subject matter or materials discussed in the manuscript (eg, employment/affiliation, grants or funding, consultancies, honoraria, stock ownership or options, expert testimony, royalties, or patents filed, received, or pending), are the following: All authors report funding of editorial support from F. Hoffmann-La Roche, Ltd. JHC has received consultancy fees from Foundation Medicine and AstraZeneca. SP has received consulting fees from Astellas, Aveo, Bristol-Myers Squibb, Eisai, Exelixis, Genentech, Ipsen, Novartis, F. Hoffman-La Roche, Ltd and Pfizer. CK and BD are employed by and own stock in Genentech. JB has received research support and grants from Pfizer; has received consulting fees and honoraria from Bristol-Myers Squibb, Genentech, Merck, Pierre Fabre, and AstraZeneca; has received royalties from UptoDate bladder.

Patient consent for publication Not required.

Ethics approval The study was conducted in accordance with the Declaration of Helsinki and International Conference of Harmonisation Good Clinical Practice guidelines, with all patients providing written informed consent before enrollment.

Provenance and peer review Not commissioned; externally peer reviewed.

Data availability statement Qualified researchers may request access to individual patient level data through the clinical study data request platform (https:// vivli.org/). Further details on Roche's criteria for eligible studies are available here (https://vivli.org/members/ourmembers/). For further details on Roche's Global Policy on the Sharing of Clinical Information and how to request access to related clinical study documents, see here (https://www.roche.com/research_and_ development/who_we_are_how_we_work/clinical_trials/our_commitment_to_ data_sharing.htm).

Open access This is an open access article distributed in accordance with the Creative Commons Attribution Non Commercial (CC BY-NC 4.0) license, which permits others to distribute, remix, adapt, build upon this work non-commercially, and license their derivative works on different terms, provided the original work is properly cited, appropriate credit is given, any changes made indicated, and the use is non-commercial. See http://creativecommons.org/licenses/by-nc/4.0/.

\section{REFERENCES}

1 Bellmunt J, Orsola A, Leow JJ, et al. Bladder cancer: ESMO practice guidelines for diagnosis, treatment and follow-up. Ann Oncol 2014;25:iii40-8.

2 NCCN Clinical Practice Guidelines in Oncology. Bladder cancer. V4, 2019. Available: https://www.nccn.org/professionals/physician_gls/ pdf/bladder.pdf [Accessed 18 Sep 2019].

3 Balar AV, Galsky MD, Rosenberg JE, et al. Atezolizumab as first-line treatment in cisplatin-ineligible patients with locally advanced and metastatic urothelial carcinoma: a single-arm, multicentre, phase 2 trial. Lancet 2017;389:67-76.

4 von der Maase H, Sengelov L, Roberts JT, et al. Long-term survival results of a randomized trial comparing gemcitabine plus cisplatin, with methotrexate, vinblastine, doxorubicin, plus cisplatin in patients with bladder cancer. J Clin Oncol 2005;23:4602-8.

5 Galsky MD, Hahn NM, Rosenberg J, et al. Treatment of patients with metastatic urothelial cancer "unfit" for cisplatin-based chemotherapy. $J$ Clin Oncol 2011;29:2432-8.

6 Surveillance, Epidemiology, and End Results Program. Cancer STAT facts: bladder cancer. Available: https://seer.cancer.gov/statfacts/ html/urinb.html [Accessed 18 Sep 2019].

7 Genentech, Inc. TECENTRIQ (atezolizumab) [package insert]. South San Francisco, CA: Genentech, Inc, 2019.

8 Roche Registration Limited. TECENTRIQ (atezolizumab) [summary of product characteristics]. Welwyn Garden City, UK: Roche Registration Limited, 2018.

9 Powles T, Eder JP, Fine GD, et al. MPDL3280A (anti-PD-L1) treatment leads to clinical activity in metastatic bladder cancer. Nature 2014;515:558-62.

10 Rosenberg JE, Hoffman-Censits J, Powles T, et al. Atezolizumab in patients with locally advanced and metastatic urothelial carcinoma who have progressed following treatment with platinum-based chemotherapy: a single-arm, multicentre, phase 2 trial. Lancet 2016;387:1909-20.

11 Petrylak DP, Powles T, Bellmunt J, et al. Atezolizumab (MPDL3280A) monotherapy for patients with metastatic urothelial cancer: long-term outcomes from a phase 1 study. JAMA Oncol 2018;4:537-44. 
12 Pal SK, Hoffman-Censits J, Zheng H, et al. Atezolizumab in platinumtreated locally advanced or metastatic urothelial carcinoma: clinical experience from an expanded access study in the United States. Eur Urol 2018;73:800-6.

13 Lee BH, Zabor EC, Tennenbaum D, et al. Renal function recovery after radical nephroureterectomy for upper tract urothelial carcinoma. World J Urol 2018;36:257-63.

14 Narita T, Hatakeyama S, Koie T, et al. Presence of transient hydronephrosis immediately after surgery has a limited influence on renal function 1 year after ileal neobladder construction. BMC Urol 2017;17:72.

15 Latcha S, Jaimes EA, Patil S, et al. Long-term renal outcomes after cisplatin treatment. Clin J Am Soc Nephrol 2016;11:1173-9.

16 Brenner BM, Meyer TW, Hostetter TH. Dietary protein intake and the progressive nature of kidney disease: the role of hemodynamically mediated glomerular injury in the pathogenesis of progressive glomerular sclerosis in aging, renal ablation, and intrinsic renal disease. N Engl J Med 1982;307:652-9.

17 Dash A, Galsky MD, Vickers AJ, et al. Impact of renal impairment on eligibility for adjuvant cisplatin-based chemotherapy in patients with urothelial carcinoma of the bladder. Cancer 2006;107:506-13.

18 Cao J, Zhao X, Zhong Z, et al. Prognostic value of pre-operative renal insufficiency in urothelial carcinoma: a systematic review and meta-analysis. Sci Rep 2016;6:35214.
19 Humphrey PA, Moch H, Cubilla AL, et al. The 2016 WHO classification of tumours of the urinary system and male genital organs - Part B: prostate and bladder tumours. Eur Urol 2016;70:106-19.

20 Alanee S, Alvarado-Cabrero I, Murugan P, et al. Update of the International Consultation on Urological Diseases on bladder cancer 2018: non-urothelial cancers of the urinary bladder. World J Urol 2019;37:107-14.

21 Klaile Y, Schlack K, Boegemann M, et al. Variant histology in bladder cancer: how it should change the management in nonmuscle invasive and muscle invasive disease? Transl Androl Urol 2016;5:692-701.

22 Hunter L, Moser J, Sturge C, et al. First-Line pembrolizumab therapy in a cisplatin-ineligible patient with plasmacytoid urothelial carcinoma: a case report. J Oncol Pharm Pract 2019.

23 Sternberg CN, Loriot $\mathrm{Y}$, James N, et al. Primary results from SAUL, a multinational single-arm safety study of atezolizumab therapy for locally advanced or metastatic urothelial or nonurothelial carcinoma of the urinary tract. Eur Urol 2019;76:73-81.

24 Kanz BA, Pollack MH, Johnpulle R, et al. Safety and efficacy of antiPD-1 in patients with baseline cardiac, renal, or hepatic dysfunction. $\mathrm{J}$ Immunother Cancer 2016;4:60.

25 Bellmunt $\mathrm{J}$, de Wit R, Vaughn DJ, et al. Pembrolizumab as secondline therapy for advanced urothelial carcinoma. N Engl J Med 2017;376:1015-26. 